\title{
Mantle cell lymphoma of the colon
}

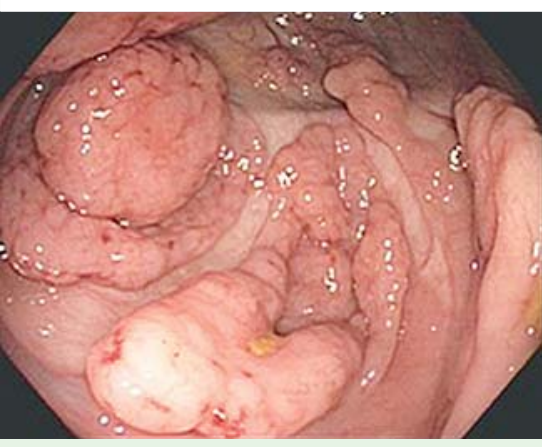

Fig. 1 Colonoscopy picture showing a 4-cm mass in the cecal base with multiple polypoid masses.

An 81-year-old man with a history of stage IV mantle cell lymphoma (MCL) diagnosed from a submental lymph node biopsy in 2006 was evaluated for new-onset melena and blood clots with bowel movements. He had been treated for his MCL with 6 cycles of CHOP-R (cyclophosphamide, doxorubicin, vincristine, prednisone, and rituximab) in 2006, followed by 13 cycles of maintenance rituximab. In 2012, he was started on lenalidomide (Revlimid), but decided to stop after an exacerbation of his heart failure.

On presentation, his hemoglobin was $5.2 \mathrm{~g} / \mathrm{dL}$. Colonoscopy showed a $4-\mathrm{cm}$ mass in the cecal base ( $\mathbf{F i g . 1}$ ), a $5-\mathrm{cm}$ ulcerated mass encircling the ileocecal valve, and six sessile odd-looking polypoid masses in the cecal base. Biopsy was consistent with MCL ( Fig.2, Fig.3, - Fig.4, Fig.5). Because of the patient's heart failure, surgical resection was not favored. He was started on radiotherapy and chlorambucil.

MCL is one of the mature B-cell nonHodgkin lymphomas. Most patients with MCL present with advanced-stage disease, and up to $80 \%$ have involvement of extranodal sites, including the spleen, bone marrow, and gastrointestinal tract. Gastrointestinal tract involvement was detected in $10 \%-28 \%$ of MCL cases in various series [1,2]. The typical appearance of intestinal MCL is multiple lymphomatous polyposis. Less commonly, it appears as protruded lesions or superficial lesions. MCL expresses pan-B-cell antigens, CD5, and FMC7. Cyclin D1 is helpful to distinguish MCL from other lymphomas.

\section{Endoscopy_UCTN_Code_CCL_1AD_2AC}

Competing interests: None
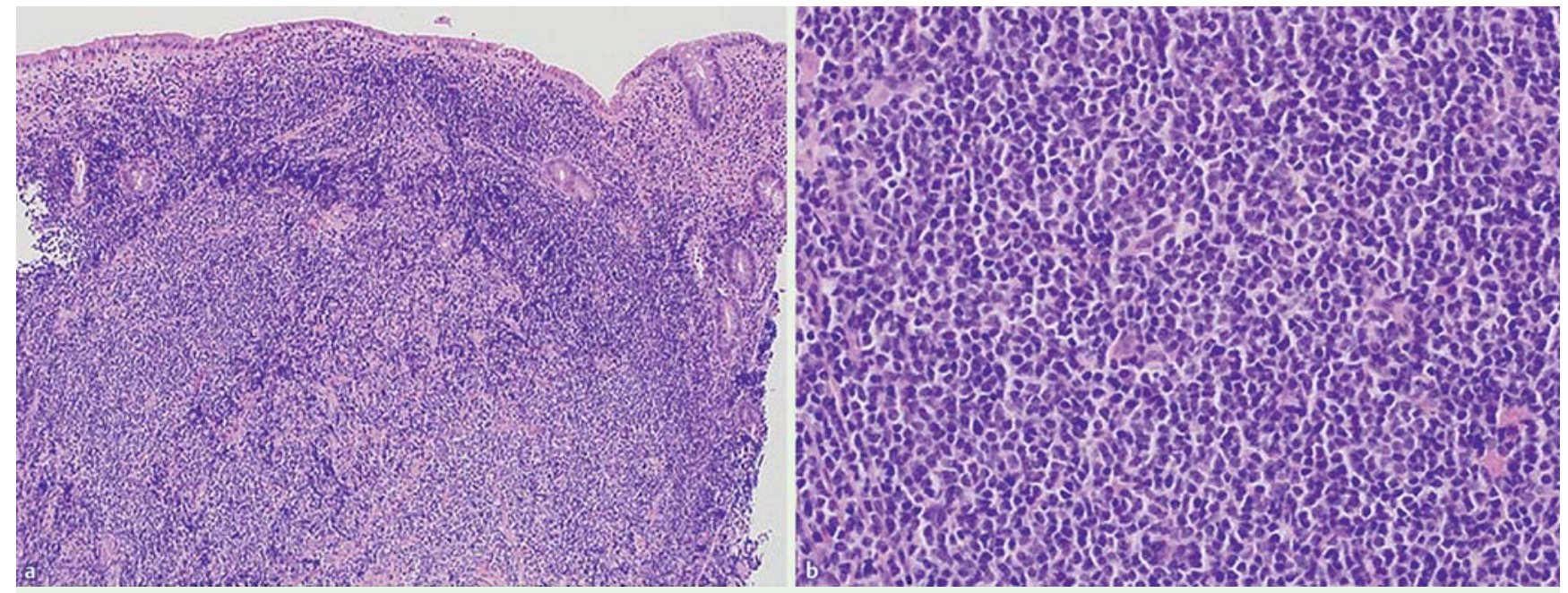

Fig. 2 a Low-power H\&E-stained section shows a diffuse lymphoid infiltrate underlying colonic mucosa. Overall, the architecture was vaguely nodular. b Higher-power H\&E-stained section shows a monotonous population of small- to intermediate-size lymphocytes with scant cytoplasm and irregular nuclear borders.

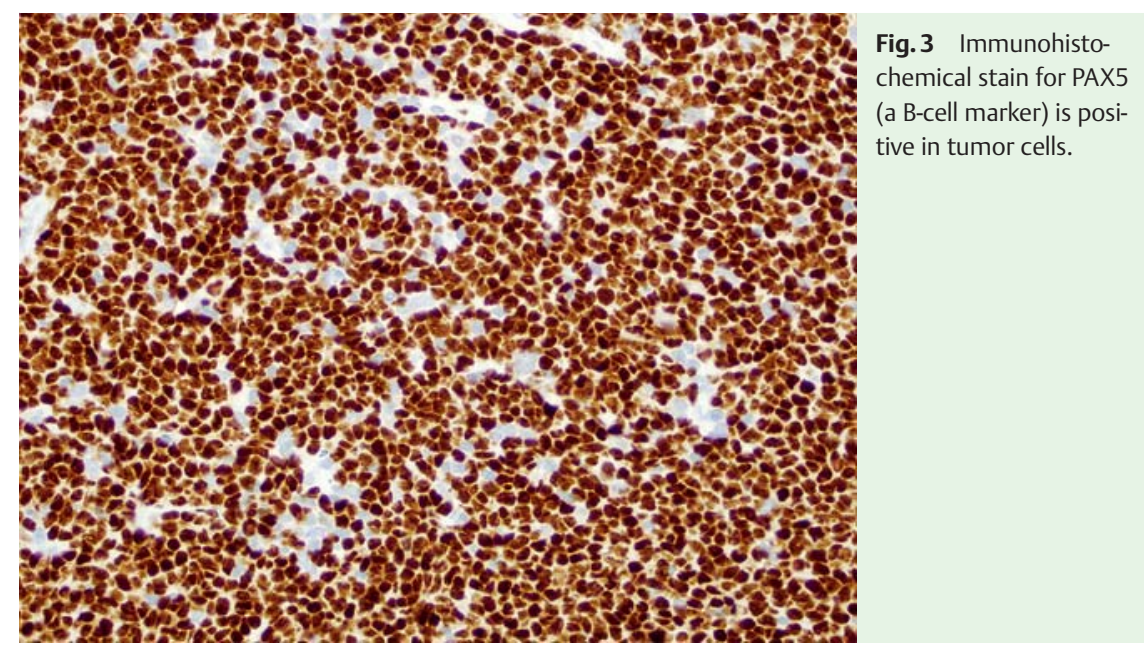



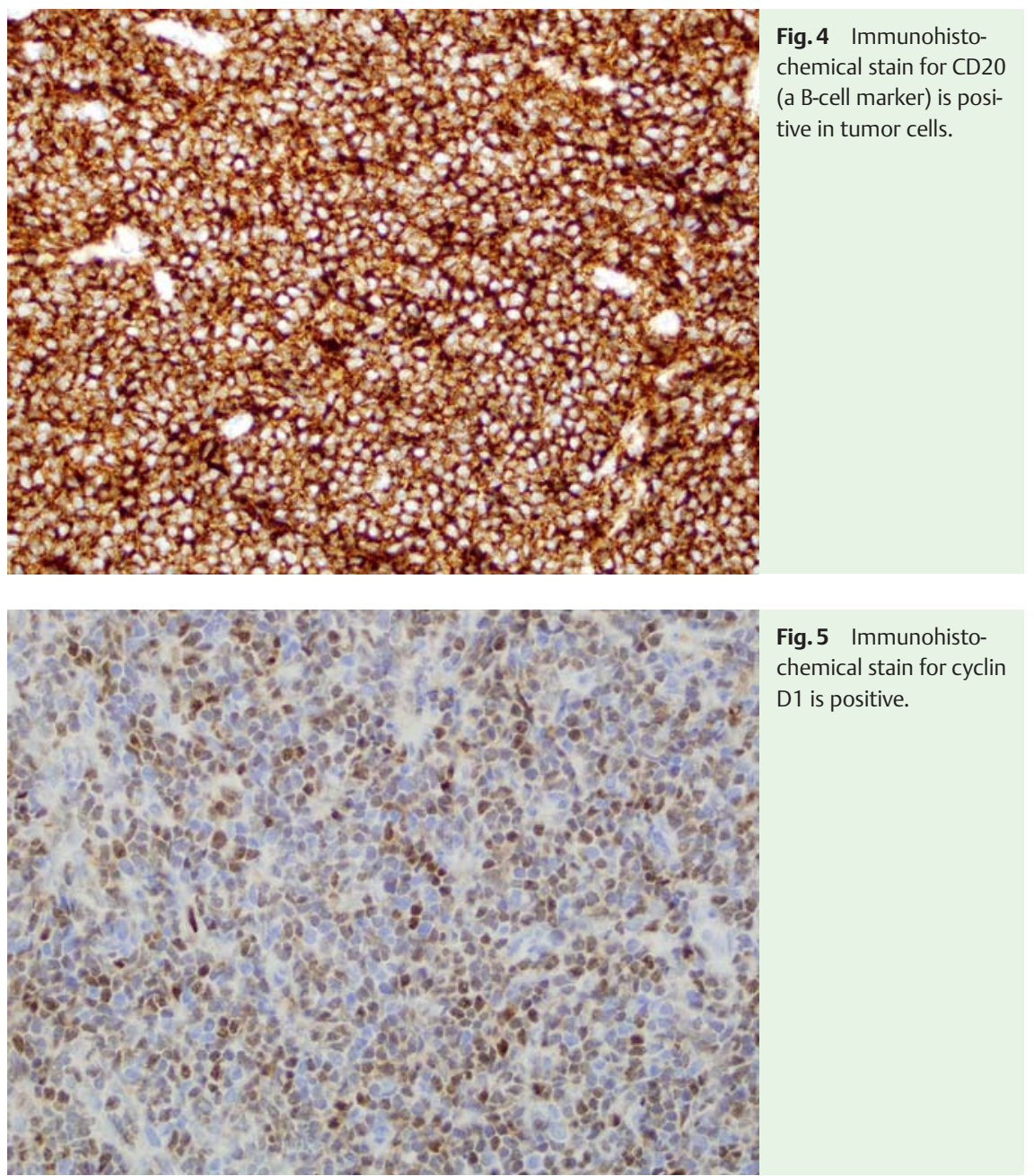

Fig.5 Immunohistochemical stain for cyclin D1 is positive.

Mohammad Esmadi, Dina S. Ahmad, Deiter J. Duff, Hazem T. Hammad

Department of Internal Medicine, University of Missouri School of Medicine, Columbia, Missouri, USA

\section{References}

$1 \mathrm{Kim} J \mathrm{H}$, Jung HW, Kang KJ et al. Endoscopic findings in mantle cell lymphoma with gastrointestinal tract involvement. Acta Haematol 2012; 127: 129-134

2 Iwamuro M, Okada H, Kawahara $Y$ et al. Endoscopic features and prognoses of mantle cell lymphoma with gastrointestinal involvement. World J Gastroenterol 2010; 16: $4661-4669$

\section{Bibliography}

DOI http://dx.doi.org/

10.1055/s-0034-1364941

Endoscopy 2014; 46: E126-E127

(C) Georg Thieme Verlag KG

Stuttgart · New York

ISSN 0013-726X

\section{Corresponding author}

\section{Mohammad Esmadi, MD}

One Hospital Dr.

DC043.00 Health Sciences Center

Columbia

MO 65212

USA

Fax: +1-573-884-5690

mohsmadi@hotmail.com 